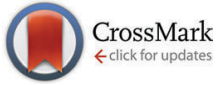

Cite this: Chem. Commun., 2015, 51, 3243

Received 27th October 2014, Accepted 9th January 2015

DOI: $10.1039 / c 4 c c 08471 c$

www.rsc.org/chemcomm

\section{On-surface self-organization of a robust metal-organic cluster based on copper(I) with chloride and organosulphur ligands $\dagger$}

\author{
Gonzalo Otero-Irurueta, $\ddagger^{\mathrm{a}}$ Irene Hernández-Rodríguez, $\ddagger^{\mathrm{b}}$ José I. Martínez, ${ }^{\mathrm{b}}$ \\ Rogger Palacios-Rivera, ${ }^{c}$ Francisco J. Palomares, ${ }^{b}$ María F. López, ${ }^{b}$ \\ Almudena I. Gallego, ${ }^{d}$ Salomé Delgado, ${ }^{d}$ Félix Zamora, $^{\text {de }}{ }^{\text {Javier Méndez }}{ }^{* b}$ and \\ José A. Martín-Gago*bc
}

\begin{abstract}
Direct sublimation of $\mathrm{C} \mathrm{Cu}_{4} \mathrm{Cl}_{4}$ metal-organic cluster on $\mathrm{Cu}(110)$ under ultra-high vacuum allows the formation of ultra-large well-organized metal-organic supramolecular wires. Our results show that the large monomers assemble with each other by $\pi-\pi$ interactions connecting dipyrimidine units and are stabilized by the surface.
\end{abstract}

The design and fabrication of new nanostructures with controlled functionality, size, shape, and position are major goals in nanoscience. ${ }^{1}$ The concept of self-assembly of molecular building blocks to generate well-defined architectures based on non-covalent interactions with the supporting substrate is technologically appealing. ${ }^{2}$ Such intriguing supramolecular assemblies often possess polymeric characteristics and are referred to as "supramolecular polymers". 3 The generation of such organized structures, obtained by controlling supramolecular interactions, makes tuning of the physicochemical properties of these molecule based materials possible. In this context, to combine organic molecules with metal entities is particularly useful. ${ }^{4,5}$ On the other hand, the bottom-up approach for forming on-surface nanostructures by direct sublimation of their building blocks under ultra-high vacuum conditions has been shown as an excellent approach to this goal. ${ }^{6}$ However, this experimental approach presents a limitation coming from the stability that it is required for the molecule to allow sublimation without structural damage. This is the reason why there are a relatively high number of nanostructures based on organization of ideal organic molecules but few of them are based on the

\footnotetext{
${ }^{a}$ Center for Mechanical Technology \& Automation-TEMA, University of Aveiro, 3810-193 Aveiro, Portugal

${ }^{b}$ Instituto Ciencia de Materiales de Madrid - CSIC, C. sor Juana Inés de la Cruz, 3, 28049-Madrid, Spain.E-mail: gago@icmm.csic.es, jmendez@icmm.csic.es

Centro de Astrobiología, INTA-CSIC, ctra. Torrejón Ajalvir s.n., 28049-Madrid, Spain

${ }^{d}$ Departamento de Quimica Inorganica, Universidad Autónoma de Madrid, UAM, E-28049 Madrid, Spain. E-mail: felix.zamora@uam.es

${ }^{e}$ Universidad Autónoma de Madrid, 28049, Madrid, Spain

$\dagger$ Electronic supplementary information (ESI) available: Synthesis, experimental and theoretical details, effect of the coverage and time, XPS results and theoretical results. See DOI: $10.1039 / \mathrm{c} 4 \mathrm{cc} 08471 \mathrm{c}$

\$ These authors contributed equally to this work.
}

combination of organic molecules with metal fragments. ${ }^{7}$ The metal-organic structures formed up to now by sublimation are almost limited to those simple cases obtained by sequential sublimation of both building blocks, organic molecules and metal precursors, or just by sublimation of the organic molecules and their subsequent in situ reaction with the metal atoms coming from the metallic surface. ${ }^{8,9}$ In both cases the selection of the building blocks has allowed formation of a large variety of $1 \mathrm{D}$ - or $2 \mathrm{D}$-coordination polymer architectures. ${ }^{10-12}$

In most of the previous studies of large complex molecules on surfaces the molecules were transferred from a solution ${ }^{13-19}$ or by a dry imprint technique ${ }^{20}$ to the substrate in order to preserve the fragile core. In this communication we focus on the search of a new metal-organic complex with a robust structure able to be sublimated keeping its molecular integrity and to self-assemble without being disrupted by the surface. We have been able to directly sublimate under ultra-high vacuum (UHV) conditions a large metal-organic cluster that is, as far as we know, the largest molecular complex ever sublimated and in situ characterized by STM. This allows the formation of well-controlled nanoarchitectures readily on a surface and use of advanced surface in situ techniques. To achieve this goal, we combine in situ scanning tunnelling microscopy (STM), X-ray photoemission spectroscopy (XPS), and low energy electron diffraction (LEED) experiments with $a b$ initio calculations.

Recently, we have reported on the synthesis and characterization of a robust metal-organic cluster $\left[\mathrm{Cu}_{4}\left(\mu_{3}-\mathrm{Cl}\right)_{4}\left(\mu-\mathrm{pym}_{2} \mathrm{~S}_{2}\right)_{4}\right]\left(\mathrm{pym}_{2} \mathrm{~S}_{2}=\right.$ dipyrimidinedisulfide) (1) showing interesting physical and chemical properties. Previous studies indicate a remarkable stability of this molecule and its capability to change its structure to produce a 2D-coordination polymer, $\left[\mathrm{Cu}\left(\mu-\mathrm{pym}_{2} \mathrm{~S}_{2}\right)(\mu-\mathrm{Cl})\right]_{n} \cdot n \mathrm{~S}\left(\mathrm{~S}=\mathrm{H}_{2} \mathrm{O}\right.$ or EtOH), when exposed to water or ethanol. ${ }^{21}$ We deposited 1 (Fig. 1a) by sublimation under UHV from a home-made Ta crucible at $370 \mathrm{~K}$ on an atomically clean $\mathrm{Cu}(110)$ surface. More details related to the sample preparation are presented in the ESI. $\dagger$

STM images (Fig. 1d) show that 1 spontaneously self-organized on the $\mathrm{Cu}(110)$ surface forming a complex molecular array. The complex structure of 1 (Fig. 1a) together with the large level of interconnection between metal-organic clusters (Fig. 1d and e) 


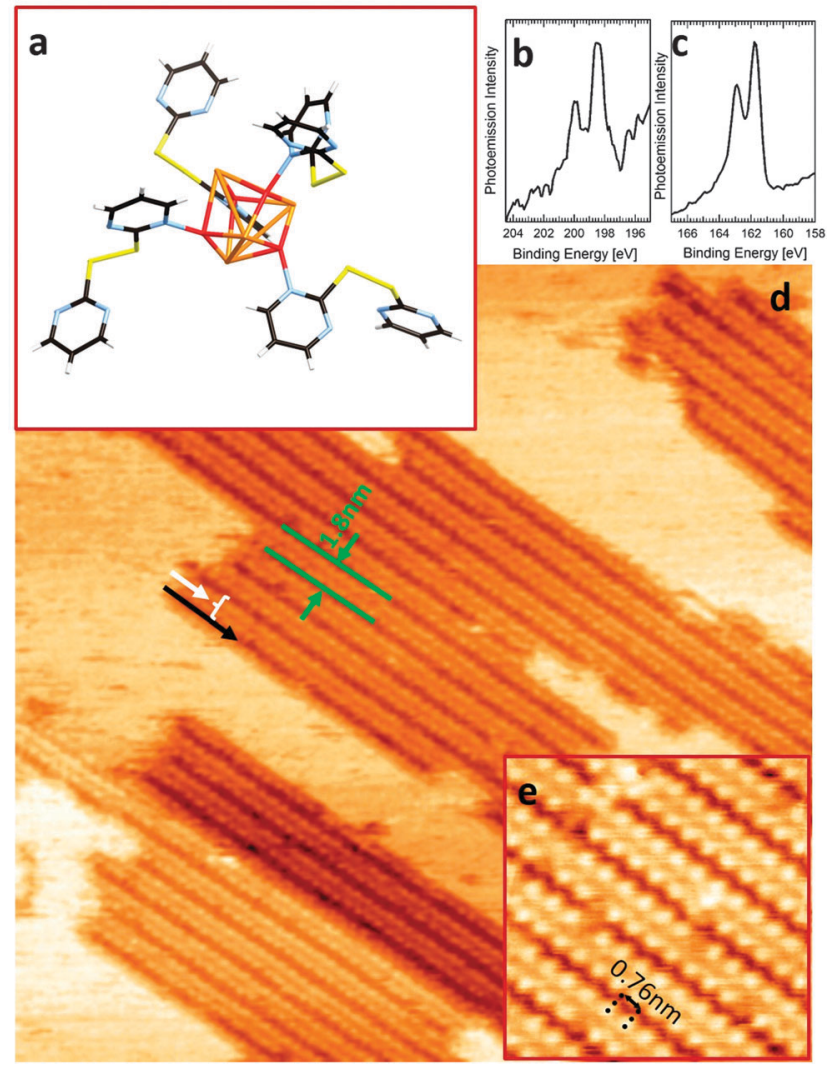

Fig. 1 (a) Model of compound 1 from DFT calculations. (b, c) Core level XPS peaks of $\mathrm{Cl} 2 \mathrm{p}$ (b), and S2p (c). STM images showing the large supramolecular assembly: (d) large scale $(50 \times 50) \mathrm{nm}^{2}, I=0.047 \mathrm{nA}, V=800 \mathrm{mV}$ and (e) highresolution $(10 \times 10) \mathrm{nm}^{2}, 0.2 \mathrm{nA}, V=-320 \mathrm{mV}$. In (a) Cu atoms are in red, $\mathrm{Cl}$ in brown, $\mathrm{C}$ in black, $\mathrm{N}$ in blue, $\mathrm{S}$ in yellow and $\mathrm{H}$ in white.

impeaches directly relating an individual molecule with any particular motif observed in the STM images. However, the observed molecular network can be described as a double-bumped row (white arrow) surrounded by dark undulating stripes (black arrow) aligned along the $[1-10]$ surface crystallographic direction. We notice that the observed morphology does not strongly depend on the bias voltage in the range of $-2000 \mathrm{mV}$ to $+2000 \mathrm{mV}$. Importantly, both surface coverage and chain length can be modulated by adjusting the deposition time (ESI†).

The distance between two consecutive dark stripes is $1.8 \mathrm{~nm}$ in the $[00-1]$ crystallographic direction while the distance between two consecutive bumps is $0.76 \mathrm{~nm}$ in the [1-10] direction. These values are in good agreement with the distance between five and three copper atoms of the substrate in the respective crystallographic directions. Furthermore, the high level of the molecular organization detected by STM is reflected in the $(5 \times 3)$ diffraction patterns obtained by LEED (ESI $\dagger$ ). These observations indicate that the supramolecular structure is not self-supported but the interaction with the metal surface underneath plays an important role in the stabilization of these macromolecular wires. On the other hand, successive STM images reveal that for intermediate coverages (ESI $\dagger$ ) the molecules can diffuse on the copper surface and part of the rows change their position. These facts indicate that the interaction with the surface is low although it cannot be completely neglected "opening the door" for the formation of different supramolecular structures on different substrates.

The chemical and structural integrity of the building blocks in the structure visualized by STM is evident after exploring the XPS results, which report binding energy values in good agreement with the expected oxidation states of the metal organic cluster (ESI $\dagger$ ). The S2p core level, Fig. 1c, shows single doublets, $S 2 \mathrm{p}_{3 / 2}$ and S2 $\mathrm{p}_{1 / 2}$, located at 161.8 and $163.0 \mathrm{eV}$, respectively. Their binding energies can be assigned to the C-S-S-C bond, ${ }^{22}$ indicating that the amino-phenyl rings are preserved. The $\mathrm{Cl} 2 \mathrm{p}$ region, Fig. 1b, consists of one doublet at 198.5 and $200.1 \mathrm{eV}$, attributable to the $\mathrm{Cl}-\mathrm{Cu}$ bonding configuration in agreement with the metal-organic nature of the molecular core.

To directly obtain the detailed structure of the observed arrays when complex molecules are involved by a simple inspection of the STM images is an impossible task. Although, considering all the experimental information together, we have carried out $a b$ initio atomistic and molecular dynamics simulations STM-DFT on different possible organizations of $\mathbf{1}$ on the surface. We have combined the localized-basis-set and plane-wave schemes as implemented in the FIREBALL ${ }^{23}$ and $\mathrm{PWSCF}^{24}$ simulation packages, respectively. In the latter, a perturbative van der Waals (vdW) correction was implemented. ${ }^{24}$ Additionally, tunnelling currents for the STM images were calculated using a Keldysh-Green function formalism, together with the firstprinciples tight-binding Hamiltonian obtained using the local-orbital DFT-Fireball method ${ }^{23,25}$ (a detailed explanation of the theoretical methods can be found in the ESI†).

We notice that the geometrical structure obtained for the gas-phase molecule changes when it is adsorbed on the $\mathrm{Cu}(110)$ surface. The structural flexibility of the legs of $\mathbf{1}$ allows its accommodation on the surface. The most significant changes affect the dipyrimidine rings of each dipyrimidinedisulfide ligand while the $\mathrm{Cu}_{4} \mathrm{Cl}_{4}$ core undergoes a readjustment in both distances and angles (ESI†). After some first geometrical discard, ab initio calculation shows that the most likely structures for the molecular chains observed in the STM images consist of molecules with the $\mathrm{Cu}_{4} \mathrm{Cl}_{4}$ core localized either on the highlighted protrusion areas or between them (dark undulating stripes observed by STM). Calculations indicate that the case is presented in Fig. 2. The bottom part of Fig. 2 shows a comparison between the simulated and experimental STM images. We show that the metallic-core of the molecule is displayed as a depression in the STM images, which mainly gives signal related to the $\pi$ electrons. The agreement is fairly good for this intricate system.

The structural and chemical integrity of the metal-organic core is preserved as it interacts neither with the surface nor with other molecules. The nature of the intermolecular interactions can be gathered by calculations. The metal-organic clusters assemble with each other forming an ultra-large supramolecular array mediated by $\pi-\pi$ interactions along the rows $([1-10]$ direction) at two different levels of height (upper panel of Fig. 2). This intermolecular bonding consists of a parallel-displaced $\pi-\pi$ stacking of all the four dipyrimidine rings. As can be seen in the upper panel of Fig. 2, both lower and higher rings pack at centroid-centroid distances of 0.33 and $0.44 \mathrm{~nm}$, which are typical values for this kind of interaction. ${ }^{26}$ 


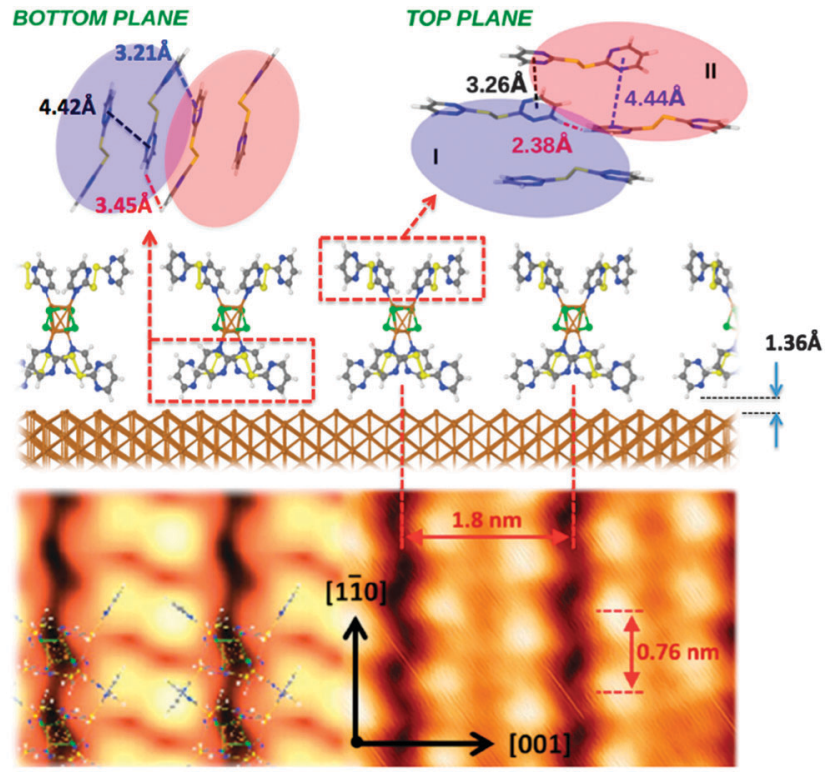

Fig. 2 Lower panel: top view of the simulated (left) and experimental (right) STM images of the self-organized compound 1 in a $(5 \times 3)$ configuration. Tunnelling parameters: $I=0.25 \mathrm{nA}$ and $V_{\mathrm{s}}=-0.75 \mathrm{~V}$. Also, the structural model is overimposed on the STM image (left). Middle panel: side view of the DFT derived model of the molecular array. Upper panel: schematic representation of the intermolecular interaction along the rows, both at the lower and upper parts of the clusters.

The interaction between the metal-organic molecular chains and the $\mathrm{Cu}$ surface, although important, is weak bonding via the $\mathrm{H}$ atoms from the most prominent ring, which suggests that the molecular chains are weakly coupled and that they preserve their own molecular properties. The substrate exclusively plays the role of a template, helping in stabilizing the structure. This is in good agreement with the high molecular diffusion observed in the experiments.

It is curious that the STM images of the rows exhibit signals between them. This suggests the existence of an intermolecular interaction in the [001] direction that can be mediated by the weak overlap caused by the extended $\pi$ electron clouds of the dipyrimidine external groups. Recently, this kind of intermolecular contrast in STM and NC-AFM images has been discussed for several systems. ${ }^{27-29}$

Occasionally, we observe small defects along the molecular array in the form of missed bumps or extra STM signals either in the dark undulating stripes or in the double bumped wires (see Fig. 1e). The most probable defect we can expect is a lack of local-order between two neighbouring molecules or a lost dipyrimidine group from the original cluster. Unfortunately, due to the complexity of the nature of the STM images it is not possible to unequivocally relate the observed features with a particular defect. However, a statistical analysis based on large area STM images over hundreds of molecules allows us to estimate the quantity of defects smaller than $5 \%$.

Interestingly, it is important to notice that the same molecular arrangement experimentally detected on $\mathrm{Cu}(110)$ may form a priori on the $\mathrm{Cu}(111)$ surface, given the degree of decoupling between the structure and the surface (see ESI $\dagger$ ).
In summary, we have shown that direct sublimation of $\mathbf{1}$ under UHV results in supramolecular rows, in which the central $\mathrm{Cu}_{4} \mathrm{Cl}_{4}$ metallic core maintains its molecular integrity. This 1D molecular macrostructure is stabilized laterally by $\pi-\pi$ intramolecular interactions and vertically via the interaction between the $\mathrm{H}$ atoms from the most prominent dipyrimidine ring and the $\mathrm{Cu}$ surface atoms. The self-assembled rows extend over the whole surface. The low interaction between the molecules and the copper substrate allows the molecular diffusion and their self-organization in large arrays covering all the substrate, suggesting that the assembled structure could structurally reversibly transform in $\left[\mathrm{Cu}\left(\mu-\mathrm{pym}_{2} \mathrm{~S}_{2}\right)(\mu-\mathrm{Cl})\right]_{n} \cdot n \mathrm{H}_{2} \mathrm{O}$ as it has been reported for the bulk compound. ${ }^{21}$ The possibility of evaporating large metal-organic units for direct self-assembling on the surface suggests a new approach to form (multi-)functional nanostructures and bring the possibility of exploring new chemical reactivity based on the knowledge of coordination chemistry.

We thank Spanish MINECO (Grants: MAT2013-46753-C2-1-P, MAT2013-47878-C2-1-R and MAT2011-26534) for financial support. JIM acknowledges a CSIC-JaeDoc fellowship, cofunded by ESF.

\section{Notes and references}

1 J. M. Lehn, Proc. Natl. Acad. Sci. U. S. A., 2002, 99, 4763-4768.

2 J. M. Lehn, Science, 2002, 295, 2400-2403.

3 L. Brunsveld, B. J. B. Folmer, E. W. Meijer and R. P. Sijbesma, Chem. Rev., 2001, 101, 4071-4097.

4 T. R. Cook, Y. R. Zheng and P. J. Stang, Chem. Rev., 2013, 113, 734-777.

5 S.-S. Li, B. H. Northrop, Q.-H. Yuan, L.-J. Wan and P. J. Stang, Acc. Chem. Res., 2009, 42, 249-259.

6 J. V. Barth, G. Costantini and K. Kern, Nature, 2005, 437, 671-679.

7 L. Vernisse, S. Munery, N. Ratel-Ramond, Y. Benjalal, O. Guillermet, X. Bouju, R. Coratger and J. Bonvoisin, J. Phys. Chem. C, 2012, 116, 13715-13721.

8 N. Lin, S. Stepanow, M. Ruben and J. V. Barth, Top. Curr. Chem., 2009, 287, 1-44.

9 J. V. Barth, Annu. Rev. Phys. Chem., 2007, 58, 375-407.

10 S. Stepanow, N. Lin and J. V. Barth, J. Phys.: Condens. Matter, 2008, 20, 184002.

11 J. Mendez, R. Caillard, G. Otero, N. Nicoara and J. A. Martin-Gago, Adv. Mater., 2006, 18, 2048-2050.

12 L. Alvarez, S. Pelaez, R. Caillard, P. A. Serena, J. A. Martin-Gago and J. Mendez, Nanotechnology, 2010, 21, 305703.

13 A. Cornia, M. Mannini, P. Sainctavit and R. Sessoli, Chem. Soc. Rev., 2011, 40, 3076-3091.

14 M. J. Rodriguez-Douton, M. Mannini, L. Armelao, A. L. Barra, E. Tancini, R. Sessoli and A. Cornia, Chem. Commun., 2011, 47, 1467-1469.

15 M. Mannini, F. Pineider, P. Sainctavit, C. Danieli, E. Otero, C. Sciancalepore, A. M. Talarico, M. A. Arrio, A. Cornia, D. Gatteschi and R. Sessoli, Nat. Mater., 2009, 8, 194-197.

16 M. Mannini, F. Pineider, P. Sainctavit, L. Joly, A. Fraile-Rodriguez, M. A. Arrio, C. C. D. Moulin, W. Wernsdorfer, A. Cornia, D. Gatteschi and R. Sessoli, Adv. Mater., 2009, 21, 167-169.

17 A. Saywell, G. Magnano, C. J. Satterley, L. M. A. Perdigao, A. J. Britton, N. Taleb, M. D. Gimenez-Lopez, N. R. Champness, J. N. O'Shea and P. H. Beton, Nat. Commun., 2010, 1, 1075.

18 A. Saywell, A. J. Britton, N. Taleb, M. D. Gimenez-Lopez, N. R. Champness, P. H. Beton and J. N. O'Shea, Nanotechnology, 2011, 22, 075704.

19 G. Otero, E. Evangelio, C. Rogero, L. Vazquez, J. Gomez-Segura, J. A. M. Gago and D. Ruiz-Molina, Langmuir, 2009, 25, 10107-10115.

20 L. Vitali, S. Fabris, A. M. Conte, S. Brink, M. Ruben, S. Baroni and K. Kern, Nano Lett., 2008, 8, 3364-3368.

21 A. Gallego, C. Hermosa, O. Castillo, I. Berlanga, C. J. Gómez-García, E. Mateo-Marti, J. I. Martínez, F. Flores, C. Gómez-Navarro, J. GómezHerrero, S. Delgado and F. Zamora, Adv. Mater., 2013, 25, 2141-2146.

22 C. Vericat, M. E. Vela, G. A. Benitez, J. A. M. Gago, X. Torrelles and R. C. Salvarezza, J. Phys.: Condens. Matter, 2006, 18, R867-R900. 
23 J. P. Perdew, Phys. Rev. B: Condens. Matter Mater. Phys., 1986, 33, $8822-8824$.

24 P. Giannozzi, S. Baroni, N. Bonini, M. Calandra, R. Car, C. Cavazzoni, D. Ceresoli, G. L. Chiarotti, M. Cococcioni, I. Dabo, A. Dal Corso, S. de Gironcoli, S. Fabris, G. Fratesi, R. Gebauer, U. Gerstmann, C. Gougoussis, A. Kokalj, M. Lazzeri, L. Martin-Samos, N. Marzari, F. Mauri, R. Mazzarello, S. Paolini, A. Pasquarello, L. Paulatto, C. Sbraccia, S. Scandolo, G. Sclauzero, A. P. Seitsonen, A. Smogunov, P. Umari and R. M. Wentzcovitch, J. Phys.: Condens. Matter, 2009, 21, 395502.
25 J. M. Blanco, F. Flores and R. Perez, Prog. Surf. Sci., 2006, 81, 403-443. 26 Y. J. Dappe and J. I. Martínez, Carbon, 2013, 54, 113-123.

27 A. M. Sweetman, S. P. Jarvis, H. Sang, I. Lekkas, P. Rahe, Y. Wang, J. Wang, N. R. Champness, L. Kantorovich and P. Moriarty, Nat. Commun., 2014, 5, 3931.

28 S. K. Hämäläinen, N. van der Heijden, J. van der Lit, S. den Hartog, P. Liljeroth and I. Swart, Phys. Rev. Lett., 2014, 113, 186102.

29 C. Weiss, C. Wagner, R. Temirov and F. S. Tautz, J. Am. Chem. Soc., 2010, 132, 11864-11865. 